\title{
Health Attitudes and Beliefs Among Hypertensive Subjects: A Study of the Hellenic Atherosclerosis Society
}

\author{
Evangelos Polychronopoulos ${ }^{1}$, Ekavi N. Georgousopoulou ${ }^{1}$, Tonia Vassilakou ${ }^{2}$, Christos Pitsavos ${ }^{3}$, \\ Christina Chrysohoou ${ }^{3}$, Vassilios Athyros ${ }^{4}$, John Goudevenos ${ }^{5}$ and Demosthenes B. Panagiotakos ${ }^{1, *}$
}

\begin{abstract}
${ }^{I}$ Department of Nutrition and Dietetics, Harokopio University, Athens, Greece; ${ }^{2}$ Department of Nutrition and Biochemistry, National School of Public Health, Athens, Greece; ${ }^{3}$ First Cardiology Clinic, School of Medicine, University of Athens, Athens, Greece; ${ }^{4}$ Second Propedeutic Department of Internal Medicine, School of Medicine, Aristotle University of Thessaloniki, Thessaloniki, Greece; ${ }^{5}$ Cardiology Clinic, School of Medicine, University of Ioannina, Ioannina, Greece
\end{abstract}

\begin{abstract}
Introduction: Health attitudes and beliefs have long been associated with lower risk of various diseases, better compliance to medication and less likelihood of relapsing. The aim of this work was to study health beliefs and attitudes of hypertensive subjects in relation to the management of cardiovascular disease (CVD) risk factors. Methods: The Hellenic Atherosclerosis Society ran a project where during 2006-2012, 10,141 individuals $(39 \pm 15$ years, 47\% men, i.e., $0.01 \%$ of the Greek population), from all areas of the country, were voluntarily enrolled and participated into a face-toface interview that gathered information about their beliefs and attitudes on diet, smoking and physical activity, as well as management of CVD disorders. Results: Self-reported prevalence of hypertension was $16.7 \%$ in men and $13.2 \%$ in women. Hypertensive subjects had lower educational level than non-hypertensive $(11 \pm 5 \mathrm{vs} .13 \pm 4.5$ years of school, $\mathrm{p}<0.001)$. Hypertensive participants as compared to normotensive seemed not to accept that unhealthy diet which consists of a major CVD risk factor $(16.2 \%$ vs. $23.1 \%$, p $<0.001)$; and they believed more in the effect of pharmaceutical medication than in healthy lifestyle. Conclusion: There is need for more effective health strategies in order to convince people, especially those with cardiometabolic disorders, on the importance of the modifiable CVD risk factors.
\end{abstract}

Keywords: Attitudes, cluster analysis, health beliefs, hypertension.

\section{INTRODUCTION}

Hypertension has been associated with the majority of cardiovascular adverse outcomes and stands as the second most important contributor to the global burden of disease, affecting almost one billion people worldwide [1]. The prevalence of hypertension varies from country to country, and in developed countries is estimated up to $20 \%$ [2-4]. Hypertension is mainly promoted by specific lifestyle behaviors, like physical inactivity, unhealthy eating, stress, as well as by specific genetic profiles [5]. The aforementioned factors have been highlighted by the most recent European Society of Cardiology guidelines that underlined the effect of a physically active life, adoption of healthier dietary habits and smoking cessation for the moderation of cardiovascular disease (CVD) risk for people with established risk factors [6].

Taking into account that lifestyle changes together with antihypertensive medication have long been established and effective on high blood pressure management, the fact that a large number of people remain undiagnosed, and of the diagnosed hypertensive subjects a large proportion remains uncontrolled, raises questions concerning the level of

*Address correspondence to this author at the Department of Nutrition and Dietetics, Harokopio University, Athens, 46 Paleon Polemiston St., 166 74, Glyfada, Greece; Tel: +30210-9603116; Fax: +30210-9600719;

E-mail: d.b.panagiotakos@usa.net awareness, as well as the impact level of the health policy strategies [4-10]. Moreover, it is known that limited knowledge and information, as well as negative health behaviors and attitudes about CVD risk factors have been associated with higher CVD risk, but also with lower likelihood of making effective lifestyle changes [7]. Contrarily, awareness and knowledge on healthy behaviors related to CVD could motivate people to make lifestyle changes and consequently reduce CVD risk [8]. Additionally, hypertensive subjects with low educational level tend to adopt negative health beliefs, which interfere in the compliance to the clinician's guidance [9]. Under this concept, the aim of the present work was to investigate the level of awareness, health attitudes and behavioral patterns of hypertensive subjects regarding CVD risk factors.

\section{METHODS}

\section{Sampling}

Between 2006 and 2012, 10,141 individuals (39 \pm 15 years, $47 \%$ men), representing $0.01 \%$ of the total population, from all areas of the country, were voluntarily enrolled. Sampling was stratified to all Greek regions based on the distribution of entire population (census 2001).

All participants were informed about the aims of the study and they gave their consent. 


\section{Measurements}

A structured, semi-quantitative questionnaire was used to retrieve the information from the participants, through faceto-face interviews, with trained investigators of the study. Based on the rationale of the study, the questionnaire included questions about the health knowledge, attitudes and beliefs of the participants. Specifically, participants were asked regarding the role of diet, physical activity and smoking, on CVD risk. In particular, they were asked: at what extent they believe that overall dietary choices may play a role in increasing CVD risk, as well as the role of olive oil, fruits and vegetables consumption (these specific food groups and patterns were especially selected because they are considered as dominant characteristics of the traditional diet of this region), nutrient supplements, sterols and stanols intake, may also decrease CVD risk. Moreover, participants were asked which components of the diet (in terms of food groups and nutrients) they believe are the most hazardous for developing CVD, and which is the safest source (i.e., individual producers, local markets, super-markets) of buying their food. In regard to smoking, the participants were asked at what extent they "truly" believe that active and passive smoking (i.e., daily exposure to environmental cigarette smoke) is responsible for cardiac events, whether daily smoking of 5 or less cigarettes is not so harmful for health, and their opinion about the effectiveness of the various antismoking therapies. The participants were also asked about their beliefs on the role of physical activity on CVD risk. In particular, they were asked: whether they knew that physical inactivity is a risk factor for CVD, and whether intensity of exercise plays a role in effectively preventing CVD. Responses were given based on a Likert-type scale (i.e., strongly disagree, disagree, neither disagree nor agree, agree and strongly agree). Moreover, age, sex, years of education (school), annual income and private home (as proxies of the financial status), and self-reported medical history of hypertension, hypercholesterolemia, diabetes, CVD and family history of CVD, together with anthropometric characteristics (i.e., weight and height), were recorded.

\section{Statistical Analysis}

Normally distributed continuous variables (i.e., age, body mass index $(\mathrm{BMI})=$ weight in $\mathrm{kg} /$ height in $\mathrm{m}^{2}$, years of education) were presented as mean values \pm standard deviation and categorical variables as frequencies. Associations between categorical variables were tested by the calculation of the chi-square test. Comparisons of mean values of normally distributed continuous variables by gender or by hypertension status were performed using the Student's t-test. Cluster analysis was also applied to reveal patterns of the participants' beliefs and attitudes regarding the aforementioned CVD risk factors. Three clusters of attitudes and beliefs were included in multiple logistic regression models, as dependent variables, and age (in years), sex (men vs. women), obesity (BMI>29.9 $\left.\mathrm{kg} / \mathrm{m}^{2}\right)$, medical history of the common CVD risk factors (yes vs. no), current smoking habits (yes vs. no), were considered as the independent predictors. All statistical analyses were performed using the SPSS version 18 (SPSS Inc., IBM Hellas, USA).

\section{RESULTS}

Self-reported prevalence of hypertension was $16.7 \%$ in men and $13.2 \%$ in women. Demographic and clinical characteristics of the participants are shown in (Table 1). The prevalence of traditional CVD risk factors was higher in hypertensive than non-hypertensive subjects, by the exception of current smoking. As it can be seen, normotensive subjects showed a completely different health status profile than hypertensive subjects (Table 1).

Almost one out of three hypertensive participants were unaware of the normal threshold of blood pressure levels (Table 2). The majority of the participants stated that they did not understand the guidelines for CVD prevention or for the management of hypertension. Participants with hypertension recognized CVD as the leading cause of mortality in a larger percentage than non-hypertensive subjects. However, all subjects underestimated the actual effect of CVD risk factors' management on CVD risk. As far as modifiable CVD risk factors are concerned (i.e., unhealthy diet,

Table 1. Socio-demographic, clinical and lifestyle characteristics of the participants $(n=10,003)$.

\begin{tabular}{|c|c|c|c|}
\hline & $\begin{array}{c}\text { Non-Hypertensive } \\
(n=8,519)\end{array}$ & $\begin{array}{c}\text { Hypertensive } \\
(n=1,484)\end{array}$ & $p$ \\
\hline Men, \% & $46.1 \%$ & $53.1 \%$ & $<0.001$ \\
\hline Education level (in years of school) & $13 \pm 5$ & $11 \pm 4.5$ & $<0.001$ \\
\hline Smoking (current), $\%$ & $37.9 \%$ & $32.3 \%$ & $<0.001$ \\
\hline Obesity $\left(\right.$ BMI $\left.>29.9 \mathrm{~kg} / \mathrm{m}^{2}\right)$ & $9.4 \%$ & $25.1 \%$ & $<0.001$ \\
\hline History of diabetes, $\%$ & $3.5 \%$ & $19.3 \%$ & $<0.001$ \\
\hline History of hypercholesterolemia, $\%$ & $6.9 \%$ & $31.8 \%$ & $<0.001$ \\
\hline History of CVD, \% & $1.6 \%$ & $14.3 \%$ & $<0.001$ \\
\hline
\end{tabular}

CVD: Cardiovascular Disease 
Table 2. Selected health attitudes of the participants, beliefs and knowledge regarding the role of diet, physical activity and smoking on CVD risk $(n=10,003)$.

\begin{tabular}{|c|c|c|c|}
\hline & $\begin{array}{c}\text { Non-Hypertensive } \\
(n=8,519)\end{array}$ & $\begin{array}{c}\text { Hypertensive } \\
(n=1,484)\end{array}$ & $\mathbf{P}$ \\
\hline Participants were unaware regarding the normal levels of arterial blood pressure & $28.3 \%$ & $38.4 \%$ & $<0.001$ \\
\hline Participants does not understand the guidelines for hypertension management and CVD prevention & $79.9 \%$ & $77.4 \%$ & 0.058 \\
\hline Participants consider CVD as the most important cause of death & $48.2 \%$ & $52.8 \%$ & 0.014 \\
\hline Participants consider unhealthy diet as the "most" important CVD risk factor & $23.1 \%$ & $16.2 \%$ & $<0.001$ \\
\hline Participants do not accept that unhealthy diet may consist a CVD risk factor at all & $29.2 \%$ & $33.6 \%$ & 0.007 \\
\hline Participants believed that the "most" important dietary habit was the food variety & $40.9 \%$ & $36.7 \%$ & $<0.001$ \\
\hline Participants believed that the "most" important dietary factor for CVD risk was saturated fat & $50.6 \%$ & $38.9 \%$ & $<0.001$ \\
\hline Participants believed that nutrient supplements, sterols and stanols cannot decrease CVD risk & $20.7 \%$ & $21.4 \%$ & 0.466 \\
\hline Participants self-reported adherence to the Mediterranean diet & $46.9 \%$ & $47.1 \%$ & 0.590 \\
\hline Participants do not accept that smoking is a major CVD risk factor & $31.2 \%$ & $33.7 \%$ & 0.212 \\
\hline Participants do not believe that physical inactivity can increase CVD risk & $61.1 \%$ & $68.6 \%$ & $<0.001$ \\
\hline
\end{tabular}

CVD: Cardiovascular disease

smoking habits, sedentary life), hypertensive and nonhypertensive subjects reported that smoking consists a major risk factor; hypertensive subjects seemed to underestimate the aggravating role of unhealthy diet and sedentary life as compared with non-hypertensive. All participants reported high level of adherence to a Mediterranean type diet, but only normotensive recognized saturated fat as an important dietary factor for CVD risk (50.6\% vs. $38.9 \%$ for hypertensive, $\mathrm{p}<0.001)$. All participants doubted that nutrient supplements, sterols and stanols could have a lowering effect in CVD risk, few of them believed that olive oil intake consumption can decrease CVD risk, with normotensive subjects being more convinced on its CVD protective effect $(17.5 \%$ vs. $19 \%$ respectively, $\mathrm{p}<0.001)$. In addition, nonhypertensive subjects tended to prefer their own or a friendly farming as their food source, more than subjects with hypertension ( $64 \%$ vs. $60 \%$ respectively, $\mathrm{p}<0.001)$.

As it was shown above, hypercholesterolemia was the most dominant cardiometabolic risk factor among hypertensive subjects. Concerning adherence to medication for the management of cholesterol levels, normotensive participants underestimated its importance, as almost $27 \%$ of them reported that they had doubts whether medication can effectively decrease cholesterol levels. In contrast, hypertensive subjects were more convinced on the lipid-lowering capacity of the suggested medication ( $41 \%$ vs. $27 \%$ of normotensive, $\mathrm{p}<0.001$ ).

Further analysis clustered participants that were characterized by the same health beliefs and attitudes into three different clusters. The first cluster was characterized by a more "lifestyle-centered" approach of CVD risk lowering.
The second cluster consisted of people who believed that medication is the most important factor for CVD risk management. Finally, the third cluster was characterized by beliefs and attitudes that implicated mainly smoking habits for CVD risk increase. Almost half (i.e., 41\%) of the hypertensive subjects were members of the second cluster, whereas $29 \%$ of hypertensive subjects belonged to the first cluster and $30 \%$ to the third. Being hypertensive it was associated with $45 \%$ (95\% Confidence Interval: $1.20,1.76)$ higher odds of belonging to the second cluster (i.e., believe in medication than any other CVD risk lowering method), independently of age, gender, smoking status, obesity, hyperlipidemia or diabetes mellitus, as compared with individuals belonging to the first cluster. No significant association was observed between the other two clusters and prevalence of hypertension.

\section{DISCUSSION}

According to the presented findings, the self-reported prevalence of hypertension was almost $17 \%$ in men and $13 \%$ in women; rates that are significantly lower than the prevalence of hypertension presented by other clinically-based studies. Moreover, hypertensive subjects seemed not sufficiently convinced for the well known role of certain lifestyle behaviors, such as sedentary life, smoking habits and unhealthy diet on CVD risk, whereas non-hypertensive subjects seemed to be more aware on their impact. In addition, hypertensive subjects tended to underestimate all modifiable lifestyle factors (i.e., physical activity, healthy diet, smoking) comparing with the non-modifiable (i.e., diabetes, hypertension, dyslipidemia). This study carries for the first time, as regards hypertensive subjects, an important public health 
message, the high level of unawareness concerning CVD risk factors and their management among hypertensive individuals. The later deserves further attention taking into account the fact that hypertension constitutes a dominant characteristic of CVD risk.

In previous findings from the same study, it was underlined that higher educational level of participants promoted adherence to healthier health beliefs. Moreover, women tend to adopt healthier beliefs and attitudes, whereas individuals with established risk factors (i.e., hypertension, diabetes mellitus, hyperlipidemia) tend to adhere to unhealthier lifestyle and adopt negative health beliefs [11].

As it was reported, the prevalence of hypertension was much lower than in other studies and in the same population. For example, the ATTICA study, in 2001, reported that the prevalence of hypertension was $38.2 \%$ in men and $23.9 \%$ in women [12]. Moreover, the majority $65 \%$ of men and $40 \%$ of women participants in the ATTICA study were untreated and only $34 \%$ of them were adequately controlled; thus, $15 \%$ of the hypertensive subjects were finally well controlled [12]. Taking also into account that the 5-year incidence of hypertension (2001-2006) in the ATTICA study was almost $14 \%$, the aforementioned rates of hypertension should be much higher in nowadays where the present work refers to [13]. Similar rates have been reported by other Greek studies, too [14]. The considerable under-reporting of hypertension status in the present work can be easily attributed to the self-reported source of the information. Nevertheless, this methodological drawback, reveals an important public health issue, that of under-diagnosis of hypertension at population level.

Unhealthy dietary habits and their role in the development of CVD seem to be underestimated, especially by hypertensive people. Although, one out of two people stated that they adhere to a Mediterranean type of diet, this finding is in discordance with previous reports in which Greek people seem to be away from the traditional dietary habits of their ancestors [15]. This discordance underlines that people have an impression of adopting healthy dietary patterns, but this is not, in fact, true. Therefore, more information and explication on healthy dietary patterns' characteristics seem to be urgently needed, especially because information and advice concerning diet could be also motivating for effective lifestyle changes, leading at the same time to a decrease of the total CVD risk [16].

In this work, it was also observed that $32 \%$ of the hypertensive participants as compared with $19 \%$ of the rest of the participants recognized salt intake as a determinant of CVD risk, mainly through the elevation of blood pressure levels. These findings are in accordance with a previous study from Switzerland, concerning the beliefs of the Swiss population on the daily recommended intake of salt and its association with hypertension. The researchers observed a high level of unawareness of the population regarding the role of salt intake, as well as the sodium content of usually consumed foods, and they concluded that health authorities should reinforce public health strategies in order to inform the population about the high impact of hypertension on CVD risk [10]. Moreover, participants doubted that nutrient supplements, as well as consumption of sterols and stanols, could have a lowering effect on CVD risk; additionally, it is surprising that only few of them believed that olive oil intake consumption can independently decrease CVD risk, with normotensive subjects being more convinced on its protective effect. It could be suggested that the use of nutrient supplements, as well as stanols and sterols' intake, in CVD risk moderation have not convinced individuals, mainly, because of the controversial findings reported in various studies [17]. Nutrient supplements, like calcium, vitamins, minerals and antioxidants, have long been investigated in relation to CVD risk, as well as to hypertension development and treatment. In particular, magnesium supplementation was suggested to improve endothelial function, vitamin $\mathrm{D}$ intake has been associated with lower CVD incidence, in observational studies, supplementation of antioxidant agents was thought to decrease CVD incidence through minimizing inflammation, omega-3 supplements have been thought to reduce blood pressure, improve endothelial function and reduce inflammation. Despite the aforementioned reports, none of the studied supplements have achieved high recommendation level, as far as CVD are concerned [18].

It has been suggested that hypertensive subjects tend to comply better to antihypertensive medication if they see an effective management of their blood pressure levels [19]. In addition, it has been shown in many studies that adherence to a healthy dietary pattern interacts with pharmaceutical treatment, leading to a better management of blood pressure levels and, consequently to a greater decrease of CVD risk [20]. This fact highlights that advice on lifestyle changes refers not only to people without diagnosed CVD risk factors, but also to people under medication as well.

As far as physical activity is concerned, it was observed that almost seven out of ten hypertensive, but also six out of ten normotensive subjects reported that they are not convinced about the role of physical activity on blood pressure levels management. At this point it should be underlined the established role of physical activity on blood pressure levels management [21, 22]; a fact that makes the aforementioned observation of major importance. The latter finding that hypertensive individuals tend to underestimate even more the role of physical activity on CVD prevention, as compared with the general population, has also been reported by other investigators too [22]. It is known that promotion of physical activity at any age group, and almost in all people, is a major strategic plan for high blood pressure control of several international organizations, and has been reported in many studies [23]. Thus, the scientific community and public health policy makers should reinforce their actions towards a more physically active lifestyle of the general population, and better inform about the health benefits of exercise on CVD prevention and its manifestations.

Concerning smoking habits, the present study reported that one out of three participants was not convinced for the harmful role of smoking on CVD risk. The proportion of people who doubt on this clearly stated issue, reveals that the existing smoking cessation campaigns have failed with a remarkable failure rate. This failure to convince has been reported also in USA, where $80 \%$ of smokers see a primary care clinician each year and approximately $70 \%$ of them want to quit smoking, suggesting that the rest $30 \%$ of smok- 
ers have not been convinced on the health damage occurring due to smoking habits. Moreover, in the same study, fewer than half of smokers have been advised to quit smoking by their primary care clinician but only $25 \%$ received evidencebased counseling and/or medication, suggesting that clinicians have failed to assess the impact of smoking habits on the health aspects of the general population. All the above mentioned studies suggest failure of the health strategies regarding smoking cessation. Thus, new, more novel and effective methods are needed to increase the percentage of smokers who receive advice to quit and assistance in quitting in the primary care setting $[24,25]$.

It was also revealed in this work a well known behavioral pattern, that of the role of physical activity and healthy diet on CVD prevention. Despite the fact that the aforementioned pattern is well known, only half of the participants seemed to adopt it. Additionally, another behavioral pattern was revealed, that of people who recognize the positive effect of medication, but do not recognize the protective role of lifestyle changes. Finally, another behavioral cluster was revealed, that of smoking which consists of the main CVD risk determinant among the lifestyle behaviors. Multi-adjusted analysis revealed that subjects who tended to believe more on medication than on lifestyle changes (i.e., second cluster) were more likely to have hypertension, as compared with the other participants belonging to the rest two behavioral patterns. To our knowledge, there is little information on health beliefs and attitudes, especially those who are lifestylerelated, among hypertensive subjects concerning the common CVD risk factors. Studying these attitudes and beliefs here, it was revealed the high level of unawareness of the population, as well as the sub-groups of people that should be targeted for informative campaigns.

\section{LIMITATIONS AND STRENGTHS}

The present study, despite its innovative ideas and the quite large and representative sample, bears several limitations. The cross-sectional design of the study cannot stand for etiological considerations. The self-report of the hypertension, as well as diabetes, hypercholesterolemic status, body weight and height, may underestimate their true prevalence; however, this gave the opportunity to compare the prevalence of self-reported medical history of the participants with known population rates, and to further discuss the level of unawareness of these conditions. To handle recall bias the interviews were performed by trained investigators.

Despite the limitations, the study is unique in its kind for the Greek population, and one of the few worldwide; it covered all regions of the country, and included the $0.01 \%$ of the total population, giving the opportunity for robust generalizations. The exploration of the predisposing factors for negative health attitudes and behaviors regarding the modifiable CVD risk factors could set the basis for public health policies in order to effectively prevent CVD in the future.

\section{CONCLUSION}

The presented findings underline the emerging need for developing a framework for policy lessons and practices against the unawareness of the role of modifiable CVD risk factors, especially under the prism of the current financial crisis. Information campaigns referring to the modifiable CVD risk factors could motivate peoples' lifestyle changes, especially for people with established CVD risk factors. Therefore, and in order to develop effective strategies to prevent the development of CVD, each community has, at first, to evaluate and understand populations' health attitudes.

\section{CONFLICT OF INTEREST}

The authors confirm that this article content has no conflicts of interest.

\section{ACKNOWLEDGEMENTS}

The authors would like to acknowledge the valuable contribution of all study's participants and the field investigators that made this project feasible. Also the authors would like to thank the Hellenic Atherosclerosis Society for supporting this project.

\section{FUNDING}

The Hellenic Atherosclerosis Society and the graduate program of the Department of Nutrition and Dietetics partially funded this project.

\section{REFERENCES}

[1] Lim SS, Vos T, Flaxman AD, et al. A comparative risk assessment of burden of disease and injury attributable to 67 risk factors and risk factor clusters in 21 regions, 1990-2010: a systematic analysis for the Global Burden of Disease Study 2010. Lancet 2012; 380: 2224-60.

[2] Wilkins K, Campbell NR, Joffres MR, et al. Blood pressure in Canadian adults. Health Rep 2010; 21: 37-46.

[3] Pereira M, Lunet N, Azevedo A, Barros H. Differences in prevalence, awareness, treatment and control of hypertension between developing and developed countries. J Hyperten 2009; 27: 963-75.

[4] Perkovic V, Huxley R, Wu Y, Prabhakaran D, MacMahon S. The burden of blood pressure-related disease: a neglected priority for global health. Hypertension 2007; 50: 991-7.

[5] James W, Van H, Shahrier A, et al. Salt-induced hypertension in a mouse model of liddle's syndrome is mediated by epithelial sodium channels in the brain. Hypertension 2012; 60: 691-6.

[6] Perk J, De Backer G, Gohlke H, et al. European guidelines on cardiovascular disease prevention in clinical practice (version 2012). Eur Heart J 2012; 33: 1635-701.

[7] Robinson JG, Fox KM, Grandy S. SHIELD Study Group: Attitudes about health and health-related behaviors in patients with cardiovascular disease or at elevated risk for cardiovascular disease. Prev Cardiol 2009; 12: 136-43.

[8] Rosenmöller DL, Gasevic D, Seidell J, Lear SA. Determinants of changes in dietary patterns among Chinese immigrants: a crosssectional analysis. Int J Behav Nutr Phys Act 2011; 8: 42.

[9] Pickett S, Allen W, Franklin M, Peters RM. Illness beliefs in African Americans with hypertension. West J Nurs Res [Epub ahead of print: 2013 Jun 13].

[10] Ditisheim A, Muradbegovic E, Bochud M, Burnier M, PechèreBertschi A. Impact of salt intake on health: beliefs of the Swiss population. Rev Med Suisse 2013; 9: 1613-6.

[11] Panagiotakos DB, Georgousopoulou EN, Polychronopoulos E, et al. Beliefs and attitudes regarding cardiovascular disease risk factors: A health survey in 10,141 Greek men and women (20062012). Int J Cardiol 2013; 168: 4847-9.

[12] Panagiotakos DB, Pitsavos $\mathrm{CH}$, Chrysohoou C, et al. Status and management of hypertension in Greece: role of the adoption of a Mediterranean diet: the Attica study. J Hypertens 2003; 21: 1483-9.

[13] Panagiotakos DB, Pitsavos C, Lentzas Y, Chrysohoou C, Stefanadis C. Five-year incidence of hypertension and its determinants: the ATTICA study. J Hum Hypertens 2007; 21: 686-8. 
[14] Athyros VG, Bouloukos VI, Pehlivanidis AN, et al. MetS-Greece Collaborative Group. The prevalence of the metabolic syndrome in Greece: the MetS-Greece Multicentre Study. Diabetes Obes Metab 2005; 7: 397-405.

[15] Tyrovolas S, Panagiotakos DB. The role of Mediterranean type of diet on the development of cancer and cardiovascular disease, in the elderly: a systematic review. Maturitas 2010; 65: 122-30.

[16] Rees K, Dyakova M, Ward K, Thorogood M, Brunner E. Dietary advice for reducing cardiovascular risk. Cochrane Database Syst Rev 2013; 3: CD002128.

[17] Genser B, Silbernagel G, De Backer G., et al. Plant sterols and cardiovascular disease: a systematic review and meta-analysis Eur Heart J 2012; 33: 444-51.

[18] Eilat-Adar S, Sinai T, Yosefy C, Henkin Y. Nutritional recommendations for cardiovascular disease prevention. Nutrients 2013; 5: 3646-83.

[19] Rajpura JR, Nayak R. Role of illness perceptions and medication beliefs on medication compliance of elderly hypertensive cohorts. J Pharm Pract [Epub ahead of print: 2013 Jul 4].

[20] Dehghan M, Mente A, Teo KK, et al. Ongoing Telmisartan Alone and in Combination With Ramipril Global End Point Trial (ONTARGET)/Telmisartan Randomized Assessment Study in ACEI
Intolerant Subjects With Cardiovascular Disease (TRANSCEND) Trial Investigators. Relationship between healthy diet and risk of cardiovascular disease among patients on drug therapies for secondary prevention: a prospective cohort study of 31,546 high-risk individuals from 40 countries. Circulation 2012; 126: 2705-12.

[21] Chomistek AK, Manson JE, Stefanick ML, et al. The relationship of sedentary behavior and physical activity to incident cardiovascular disease: Results from the Women's Health Initiative. J Am Coll Cardiol 2013; 1097: 1404-6.

[22] Kokkinos P, Panagiotakos DB, Polychronopoulos E. Dietary influences on blood pressure: the effect of the Mediterranean diet on the prevalence of hypertension. J Clin Hypertens 2005; 7: 165-70.

[23] Huai P, Xun H, Reilly KH, et al. Physical Activity and Risk of Hypertension: A meta-analysis of Prospective Cohort Studies. Hypertension [Epub ahead of print: 2013 Sep 30].

[24] Piccini RX, Facchini LA, Tomasi E, et al. Promotion, prevention and arterial hypertension care in Brazil. Rev Saude Publica 2012; 46: 543-50.

[25] Piper ME, Baker TB, Mermelstein R, et al. Recruiting and engaging smokers in treatment in a primary care setting: developing a chronic care model implemented through a modified electronic health record. Transl Behav Med 2013; 3: 253-63.

Received: October 31, 2013

Revised: November 12,2013

Accepted: November 12, 2013

(C) Polychronopoulos et al.; Licensee Bentham Open.

This is an open access article licensed under the terms of the Creative Commons Attribution Non-Commercial License (http://creativecommons.org/licenses/by-nc/3.0/) which permits unrestricted, non-commercial use, distribution and reproduction in any medium, provided the work is properly cited. 